\title{
Trans-ophthalmic Arterial Ethanol Embolotherapy for Arteriovenous Malformations: A Single Centre Experience
}

\section{Xin-yu Li}

Shanghai Jiao Tong University School of Medicine

Xi-tao Yang ( $\nabla$ xitao123456@126.com)

Shanghai Red Cross Ninth People's Hospital: Shanghai Jiao Tong University School of Medicine Affiliated Ninth People's Hospital https://orcid.org/0000-0003-4030-4394

\section{Research Article}

Keywords: Arteriovenous malformations, Ophthalmic artery, Transarterial Embolization, Ethanol

Posted Date: October 27th, 2021

DOI: https://doi.org/10.21203/rs.3.rs-1016605/v1

License: (c) (i) This work is licensed under a Creative Commons Attribution 4.0 International License. Read Full License 


\section{Abstract}

Background and purpose

Arteriovenous malformations (AVMs) derived ophthalmic artery (OphA) branches are not common, however, their management is very challenging. We aimed at evaluating the safety and efficacy of Trans OphA ethanol embolotherapy for these lesions.

Materials and methods

We retrospectively reviewed 26 patients with AVMs fed by OphA, who underwent transOphA embolization using ethanol from February, 2015 to December, 2019. Sixty-six transOphA embolotherapy procedures (range, 1-4 procedures; mean, 2.5 procedures) were performed. Degree of devascularization, visual field, visual acuity, and quality-of-life outcomes were compared and analyzed at follow ups (mean, 32.6 months; month range 10-60). Complications were recorded.

Results

Twenty of the 26 patients (77\%) reported complete or $>90 \%$ AVM devascularization while six patients $(23 \%)$ showed $>70 \%$ devascularization. Eleven patients (42\%) presented with visual acuity impairments with 4 complete relief, 6 improvements. Eight patients (42\%) presented visual field defects with 4 complete relief, 3 with improvements. Ten patients (38.4\%) presented with diplopia and exophthalmos with 2 complete relief, 6 major improvements. Bleeding was controlled in all cases $(100 \%)$. All patients (100\%) exhibited cosmetic deformities with 17 being completely relieved. Moreover, all patients $(100 \%)$ exhibited impaired daily life, which was resolved in 21 patients with 5 patients reporting major improvements. No vision loss, death, or permanent disability in all patients.

\section{Conclusions}

TransOphA ethanol embolotherapy was found to be efficacious, safe and it achieved symptomatic resolution or improvement of AVMs fed by OphA with acceptable complications without the risk of visual impairment.

\section{Introduction}

Arteriovenous malformations (AVMs), which mainly derived from ophthalmic artery branches (OphA) cause the periorbital disfigurement, ulcer, bleeding, ocular and visual functional problems ${ }^{4,7,18}$. Surgical ligation of feeding arteries or partial excision of AVM lesions can potentially induce the recurrence or worsening symptoms ${ }^{11}$. Endovascular interventions have been used to treat AVMs that have a high risk of surgery and/or surgically inaccessible lesions, especially in the periorbital and orbital regions ${ }^{12,25}$. TransOphA embolization using embolic agents (PVA particles, Gelfoam powder, n-Butyl-2Cyanoacrylate (NBCA) Glue, and Onyx) has been reported to be successfully used to treat various vascular malformations and neoplastic lesions fed by OphA, including intraorbital arteriovenous fistulas ${ }^{4}$, AVMs in craniofacial regions ${ }^{3}$, and meningiomas 28 15, 29 . In AVMs fed by OphA, targeting of these aforementioned embolic agents to the nidus is difficult. In addressing the limitations associated with low dispersion of embolic agents, ethanol embolotherapy has shown positive outcomes in AVM treatment with acceptable complication risks ${ }^{22}$. In the presence of high risks anatomical areas where the central retinal artery arises off of the ophthalmic artery, reflux of embolic agents into this vessel could result in permanent blindness. Endovascular embolization of AVMs fed by OphA is less well described in the literature and has been historically limited by the technical challenges of transarterial embolization via the ophthalmic artery and the perceived risk of vision loss. Based on an in-depth understanding of the angioarchitecture of AVMs, approaches, materials, and treatment results over several years, one would expect that ethanol endovascular treatment modalities and principles would also evolve. 
At present, we aimed at assessing the efficacy of transarterial embolization for AVMs fed by OphA using ethanol embolotherapy. This study was approved by the Scientific Research Projects Approval Determination of Independent Ethics Committee. Informed consents for procedures were obtained from all study participants.

\section{Materials And Methods}

Retrospective assessment of medical records and radiologic images was approved by the Institutional Review Board of the hospital. Medical records for all patients with high-flow AVMs in a database of 86 patients with AVMs fed by OphA were retrospectively reviewed. A total of 26 patients presenting with transarterial embolization between February, 2015 and December, 2019 were enrolled for analysis in this study. At the time of treatment, average age of the 26 patients (17 male, 9 female) was 32.3 years (range, 16-57 years). The 26 patients presented AVMs at different locations: nose $(n=3)$, eye lid $(n=20)$, and orbital $(n=3)$ (Table 1). 23 patients $(77 \%)$ had previously received inappropriate treatments, including embolization or surgical ligation of the external carotid artery branches $(n=18)$, incomplete surgical resection $(n=16)$, and percutaneous puncture ethanol sclerotherapy $(n=6)$ in another institutions, which induced lesion expansion and deterioration (Table 1). Fourteen (54\%) of the 26 patients reported that AVM had been diagnosed at birth with 8 (31\%) patients showing obvious lesion enlargement during pregnancy $(n=5)$ or injury $(n=3)$.

Patients had presented with various signs and symptoms, including pain, pulsating mass, swelling, reduction of the visual field, deteriorated visual acuity, bleeding, and skin ulcerations. Clinical data from pretreatment imaging studies as well as ocular and visual symptoms (acuity measurement and visual field test) for every patient were retrieved.

\section{Endovascular procedures}

Endovascular procedures were performed under general anesthesia with saline-flushed catheters under routine heparinization. To assess angioarchitectures of AVMs, including feeding arteries, draining veins, nidus of AVMs, angiograms for both internal and external carotid arteries were obtained.

Vascular access was achieved through right common femoral artery. The $5 \mathrm{~F}$ guiding catheter was placed at the $\mathrm{C} 2$ segment of the internal carotid artery after which an angiogram was initiated. In all cases, there were the hypertrophy of OphA branches, indicating transOphA embolization was feasible. Choroidal blush and safety point [origin of central retinal artery (CRA)] were identified on the angiogram (Fig. 1). Then, a 1.7F microcatheter (ev3) was navigated by 0.014 microguide wire (Bonston) into a branch of OphA and positioned distal to origin of the central retinal artery to minimize reflux. The aim was to place the tip of the microcatheter super selective in the nidus or as close as possible to it with both inflow and outflow zones (distal part of feeding artery/arteries and proximal part of draining vein) (Fig. 2). Then, the contrast medium was injected while avoiding its back streaming to the proximal part of the ophthalmic artery before ethanol injection under fluoroscopy. Since ethanol is radiopaque, its reflux was indicated by reflux of the contrast medium. Reflux of the contrast medium over the microcatheter indicated a decrease in the speed of ethanol injection.

After several test injections, the amount of ethanol used during embolization was based on the amount of contrast medium required to fill the portion of AVMs being treated by manually injected ethanol. Arteriography was performed 5-10 min after each ethanol injection to determine whether blood flow stasis had been achieved. Then, embolization was performed to obliterate all or part of nidus until all the desired clinical results were achieved (Fig. 3). Coils were used in 3 patients. For these cases, coils (Nester; Cook Medical, Bloomington, Ind) were first deployed to decrease the blood flow in the nidus via direct puncture approach. Next, the ethanol was injected.

\section{Evaluation of clinical data and follow-up results}

Data on symptoms and quality of life (QoL) at follow up (through telephone conversations) was obtained by completion of questionnaires assessing specific symptoms before and after endovascular treatment. For patients whose treatments were 
complete, telephone questionnaires and physical examinations were performed at 1- and 3-month intervals, respectively, after last procedures. Clinical follow-ups were done by phone within 2 weeks of embolization followed by 4-week clinical visits for follow-ups. The assessed symptoms included pain, visual status (vision acuity and visual field), cosmetic deformity, and impairments of daily life activities. Patient symptoms before and after embolization were compared using a 5 -point scale as previously reported ${ }^{17}$ : 1 , worsening; 2 , no change; 3 , minor improvements; 4 , major improvement; and 5 , complete relief based on previous reports. major improvement; and 5, complete relief. Major improvement and complete relief were considered as significant improvement after embolization. In addition, the occurrence of any side effects or complications and satisfaction associated with treatment were documented.

Assessments of radiological images during follow-up was achieved by comparison of final angiograms by three experienced interventional neuroradiologists. Results of post-treatment digital subtraction angiography (DSA) were interpreted as: $1,<50 \%$ shunt reduction; $2,>50 \%$ shunt reduction; $3,>70$ shunt reduction; 4 , >90\% shunt reduction; and 5 , complete AVM occlusion. The detected complications, classified as minor or major, in relation to the embolization procedure were then analyzed ${ }^{5}$.

\section{Statistical analysis}

Statistical analyses were performed using SPSS V.21.0 (SPSS, Chicago, Illinois, USA) and SAS 9.2 (SAS Institute, Cary, North Carolina, USA). Paired nominal data were matched with the Mc-Nemar test to determine the significance of differences between pre-embolization and post-embolization angiography and clinical long-term results. $p<0.05$ was considered statistically significant. Paired-samples t test was used to calculate the difference in numeric variables (such as visual acuity) pre- and posttreatment. Snellen acuity was converted to logarithm of minimal angle of resolution values.

\section{Results}

A total of 66 embolization procedures (range 1-4, mean 2.5) were performed on the 26 patients. Majority of the patients were treated in multiple stage embolization sessions. One embolization session was performed on 1 patient, two sessions on 13 patients, and more than three sessions on 12 patients. The mean volume of injected ethanol in each procedure was $5.9 \mathrm{ml}$ (range 0.6-11.5 ml), whereas mean follow-up time was 32.6 months (range 10-60). Notably, $77 \%$ of patients had $100 \%$ closure of AVM (Fig. 3) or $57.7 \%$ had $>90 \%$ closure of AVM angiographically confirmed while one patient had $>50 \%$ closure of AVM (Table 1).

\section{Visual outcomes}

Visual outcomes were summarized Table 2. Prior to embolization, 11 patients (42.3\%) had a deficit in visual acuity, which experienced complete relief in $36.6 \%$ of patients, major improvement in $27.2 \%$ of patients, minor improvement in $27.2 \%$ of patients, and unchange in $9 \%$. All patients without preoperative visual deficit remained unchanged $(p<0.01 ;$ Fig. $4 a)$. With regard to the visual field, 8 of all 26 patients (30.7\%) showed impairment. Of these 8 patients, $50 \%$ had complete relief, $25 \%$ major improvement, $12.5 \%$ minor improvement, and $12.5 \%$ no change with regard to visual field deficits. In contrast, all patients without preoperative visual field impairment remained unchanged ( $p<0.01$; Fig. 4b).

Ten patients (38.4\%) presented with diplopia and exophthalmos; 2 were completely relieved, 6 had major improvements while 2 had minor improvements. Worsening of functions was not observed.

\section{QoL questionnaire}

Questionnaires from the 26 patients were included in the evaluation (Table 2). Bleeding as well as levels of pain, cosmetic deformities, and impairments in daily life before and after endovascular treatment were recorded and statistically analyzed. 
Statistically significant differences $(\mathrm{p}<0.05)$ in pain or bleeding between pre-embolization and post-embolization patients were observed. Pain was resolved in $6(23 \%)$ patients and was significantly improved in $1(4 \%)$ patient. Bleeding was controlled in 12 (46\%) cases with none reporting any recurrent pain or hemorrhage due to AVM after endovascular treatment. All patients presented cosmetic concerns, which were main reasons for seeking intervention. After treatment, 17 (65.4\%) patients had complete relief of cosmetic deformities while 1 still showed minor cosmetic deformities. Eight (30.8\%) of the patients had clearly visible improvements in their cosmetic deformities after endovascular treatment.

The 26 patients (100\%) reported impairments in their daily lives, which were directly related to AVM. Twenty-one (80.8\%) of the patients had no impairments in their daily lives after treatment with $5(19.2 \%)$ of the patients experiencing major improvements. There were no impairments in daily life after endovascular treatment.

\section{Complications}

There were no procedure-related vision loss, stroke, permanent disability, or death. Ten (38.5\%) of the patients developed complications. These complications included two cases with concurrent of superficial skin necrosis and kin bulla, and four cases of skin bulla alone. Wound dressing healed all superficial skin necrosis and bullae cases, and there were no further invasive treatments, including skin grafts that were needed. Three cases presented with blurry vision that was attributed to spasm of central retinal artery after transarterial embolization. They had resolved completely after embolization with lowmolecular weight heparin (q12 hours for the first 72 hours). One case of transient nerve injury, which presented with hyperalgesia in the frontal region, spontaneously resolved with conservative treatment within one month.

\section{Discussion}

AVMs derived from OphA are rare, with limited relevant reports on treatment outcomes. Our findings show that transOphA ethanol embolotherapy is an effective and safe treatment modality in the series. We established that transOphA ethanol embolotherapy alone achieved satisfactory clinical and imaging outcomes for these lesions with accepted complications and no procedures-related permanent vision impairment. For cases with visual impairment caused by AVMs, a higher rate of postembolotherapy visual recovery was achieved.

Previous studies reported that treatment of AVMs fed by OphA is clinically challenging due to complex hypervascularity of AVMs and the risk of vision loss from OphA involvement $1,4,21$. Endovascular treatment is effective for AVMs $5,8,24$, and ethanol embolotherapy of periorbital and orbital AVMs improves symptoms to some extent $14,15,25$, and yet the overall results are not satisfactory. However, due to its close proximity to retinal central artery, which regulates visual acuity and extraocular movements, therapeutic approaches that are specific for treatment of AVMs fed by OphA are scarce.

Accessibility to AVM nidus comprises transarterial, transvenous or direct puncture approaches, but there is no consensus about which is best. Percutaneous ethanol sclerotherapy extravasates into surrounding tissues resulting in catastrophic complications, including ocular infarctions or reversible blurred vision ${ }^{14}$. Su et al. ${ }^{25}$ reported that one AVM patient in the periorbital region received percutaneous ethanol sclerotherapy and developed a blurred vision. Transvenous embolization is reserved for lesions with a venous sac ${ }^{24}$, which was absent in the cases included in this study. Twelve (46\%) of the patients in this study who underwent ethanol sclerotherapy via the direct puncture approach failed to control intractable epistaxis, progressive visual or ocular symptoms. Therefore, it is necessary to try transarterial embolotherapy for AVMs fed by OphA. From an anatomical point of view, hypertrophy of OphA branches in the present cohort provided the ideal access to nidus via transOphA approach. In terms of these points, we preferred to use transOphA approach as access to nidus of choice for the treatment of AVMs.

Due to risks of visual and extraocular complications, trans-OphA embolization has been considered to be a less-thanoptimal strategy ${ }^{15}$. Visual complications originating from transarterial embolization of lesions using a liquid embolic agent include palpebral and orbito-frontal arteriovenous fistulas, and meningiomas of skull base ${ }^{28}$. Terada et al. reported

Page 5/12 
that one out of four cases of embolization of meningiomas (through OphA branches) experienced postembolization visual deficits ${ }^{26}$. Furthermore, Lv et al. reported that 3 pure intraorbital arteriovenous fistula patients underwent transarterial embolization using Onyx, leading to permanent blindness due to Onyx reflux and occlusion of the central retinal artery ${ }^{15}$. An adult man experienced multiple branch arterial occlusions resulting in severe visual field defects after treatment of eyelid AVM. Visual deteriorations rate account for the reflux of embolic agents ${ }^{23}$.

To avoid the reflux, the understanding of anatomic safe points of OphA and hemodynamics facilitated the achieve of successful and safe embolization. In treating AVMs fed by OphA, neurointerventionalists must consider anatomic safe points of OphA and hemodynamics. It is advisable to carefully distally position the microcatheter tip. "Safety point" is recognized on angiogram, and is considered as a secure limit for embolization beyond safety points with a minimal risk of visual deficits ${ }^{1}$. These precautions guarantee the preservation of the central retinal artery. As a result, all patients were exclusively treated with ethanol injection via OphA without procedural visual impairments. In addition, previous studies have concluded that since embolized defective blood vessels can redirect and transport embolic material to unintended locations, changes in blood flow correlate with visual complications ${ }^{19}$. Therefore, multiple staged embolizations and low ethanol volumes in each session might contribute to a reduced risk of reflux, leading to low complications.

The choice of embolic agents (ethanol) depends on effectiveness and safe profile. The available embolic agents including PVA particles, Onyx, and gluehave a limited distal access to nidus ${ }^{13}$, especially in cases of long distances between microcatheter tip and point of shuntsEthanol achieved the desired embolic effect in addressing the long distance embolization because of its high fluidity and a more distal lesional penetration ${ }^{22}$.Ethanol is also reported to be the best agent to achieve endothelial ablation of the AVM nidus ${ }^{24}$. It achieves potential cure by causing fracture up to the internal elastica lamina of the vascular walls, eliminating angiogenesis factors, eventually leading to permanent occlusion of the exposed vessels and prohibiting further neovascularization ${ }^{24}$. The main goal of AVMs was to eradicate shunts, which induced OphA-related risks and symptoms including bleeding, pain, vision impairment, and cosmetic deformities. In this study, $100 \%$ closure of AVM was achieved in 20 (77\%) patients, which was higher than the results of previous studies that reported nearly complete obliteration with Onyx in eight of 18 (44.4\%) cases ${ }^{27}$, and polyvinyl alcohol particles or NBCA in $10(14.5 \%)$ of patients, even $58.4 \%$ by combined surgical and presurgical embolization ${ }^{8}$. AVMs cause visual impairment or visual loss via arterial steal ${ }^{2,10}$. In the cohort, eleven patients (42.3\%) presented with visual acuity impairments with 3 complete relief, 6 improvements. Despite that ischemic time is reported to be an indicator of poor prognosis of vision improvement ${ }^{14}$, our study clearly showed eradicating arteriovenous malformation using ethanol embolotherapy facilitated improvement of visual outcomes in the cases with vision impairment. The potential cause for the improved visual function may be the increased perfusion of retina after obliterating the fistulas and reducing arterial steal.

The procedural visual impairment is a complication worthy of special attention. Three (11.5\%) of the 26 patients in this study had visual deteriorations, which was similar compared to findings from previous studies, which reported $8 \%-30 \%$ visual deterioration rates using embolization with Onyx as the main approach ${ }^{4,16}$. Visual deteriorations may be attributed to thromboembolism or unintentional obstructions of the CRA ${ }^{6}$. Notably, these three cases spontaneously resolved completely 24 hours after conservative treatment without the residual permanent impairment. We hypotheses that ethanol concentrations in the feeding artery are reduced in the reflux process with degree of ethanol damage reduced.

The most common complications reported skin necrosis owing to over injection and reflux of ethanol ${ }^{4,9}$, especially in the periorbital skin. Park et al. reported that intra-arterial ethanol injection causes skin necrosis because tiny, angiographically invisible normal arteries become exposed to ethanol. Minimizing intra-arterial ethanol injections contribute to reduction of overall complication rates ${ }^{20}$. Majority of AVMs fed by OphA in this study were in the superficial skin of the eyelid. Eyelid necrosis which spontaneous healed was observed in this study. Therefore, close patient monitoring of these adverse events and complications following ethanol sclerotherapy is necessary. In addition, ethanol may exert its effects to sites that are 
distant from the target site, including the venous system, thereby causing thrombophlebitis, hemoglobinuria, cardiopulmonary collapse, and death, which were not observed in this study.

This study has some limitations. First, this was a retrospective, single-center study with a relatively small sample size that was attributed to the rarity of AVMs fed by OphA. Second, being a fairly uncommon disease with relatively small number of patients, inherent selection biases due to observational designs and monocentric nature of the current study could have affected the findings.

\section{Conclusions}

Ethanol for transarterial embolotherapy of AVMs fed by OphA showed positive outcomes for improvement of clinical symptoms, including visual functions and cosmetic deformities with acceptable complications. Transarterial embolotherapy might be advocated. Staged embolizations were found to be safe and clinically effective based on understanding of "safety point" and injection rate. TransOphA ethanol embolization is recommended as the mian routine treatment of AVMs fed by OphA.

\section{Declarations}

Funding $\mathrm{T}$ This study received Fundamental research program funding of Ninth People's Hospital affiliated to Shanghai Jiao Tong university School of Medicine (No. JYZZ076), Clinical Research Program of Ninth People's Hospital, Shanghai Jiao Tong University School of Medicine (No. JYLJ201801, JYLJ201911), the China Postdoctoral Science Foundation (No. 2017M611585) and the National Natural Science Foundation of China (No. 81871458).

Conflicts of interest/Competing interests[no conflicts of interest.

Availability of data and material[Additional data and material are available upon request

Code availabilityロNot applicable

Ethics approval[All procedures performed in the studies involving human participants were in accordance with the Scientific Research Projects Approval Determination of Independent Ethics Committee of Shanghai Ninth People's Hospital, Shanghai JiaoTong University School of Medicine, with the 1964 Helsinki Declaration and its later amendments or comparable ethical standards.

Consent to participate】All patients provided signed written informedconsent to participate.

Consent for publication: All authors consent for publication.

Authors' contributions: Xi-tao Yang designed experiments; Xin-yu Li carried out experiments, and wrote the manuscript, Xitao Yang performed manuscript review.

\section{References}

1. Alvarez H, Rodesch G, Garcia-Monaco R, Lasjaunias P. Embolisation of the ophthalmic artery branches distal to its visual supply. Surg Radiol Anat 1990;12:293-297.

2. Andracchi S, Kupersmith MJ, Nelson PK, Slakter JS, Setton A, Berenstein A. Visual loss from arterial steal in patients with maxillofacial arteriovenous malformation. Ophthalmology 2000;107:730-736.

3. Aoki S, Sasaki Y, Machida T, Sasaki Y. Embolization of extensive facial arteriovenous malformation via the orbital branches of the ophthalmic artery. Radiat Med 1991;9:98-100. 
4. Caragine LP, Jr., Halbach VV, Dowd CF, Higashida RT. Intraorbital arteriovenous fistulae of the ophthalmic veins treated by transvenous endovascular occlusion: technical case report. Neurosurgery 2006;58:ONS-E170; discussion ONSE170.

5. $\quad$ Cardella JF, Kundu S, Miller DL, Millward SF, Sacks D, Society of Interventional R. Society of Interventional Radiology clinical practice guidelines. J Vasc Interv Radiol 2009;20:S189-191.

6. Chronopoulos A, Schutz JS. Central retinal artery occlusion-A new, provisional treatment approach. Surv Ophthalmol 2019;64:443-451.

7. Clarencon F, Blanc R, Lin CJ, et al. Combined endovascular and surgical approach for the treatment of palpebral arteriovenous malformations: experience of a single center. AJNR Am J Neuroradiol 2012;33:148-153.

8. Dmytriw AA, Ter Brugge KG, Krings T, Agid R. Endovascular treatment of head and neck arteriovenous malformations. Neuroradiology 2014;56:227-236.

9. Fernandez-Alvarez V, Suarez C, de Bree R, et al. Management of extracranial arteriovenous malformations of the head and neck. Auris Nasus Larynx 2020;47:181-190.

10. Fritz W, Klein HJ, Schmidt K. Arteriovenous malformation of the posterior ethmoidal artery as an unusual cause of amaurosis fugax. The ophthalmic steal syndrome. J Clin Neuroophthalmol 1989;9:165-168.

11. Gilbert P, Dubois J, Giroux MF, Soulez G. New Treatment Approaches to Arteriovenous Malformations. Semin Intervent Radiol 2017;34:258-271.

12. Hou K, Wu W, Liu Y, Qu L, Xu B, Yu J. Role of the ophthalmic artery in the endovascular treatment for intracranial vascular diseases. Acta Neurol Belg 2021;121:321-330.

13. Hu J, Albadawi H, Chong BW, et al. Advances in Biomaterials and Technologies for Vascular Embolization. Adv Mater 2019;31:e1901071.

14. Link T, Kam Y, Ajlan R. Ocular infarction following ethanol sclerotherapy of an arteriovenous malformation. Am J Ophthalmol Case Rep 2019;15:100457.

15. Lv X, Li W, Liu A, Lv M, Jiang C. Endovascular treatment evolution for pure intraorbital arteriovenous fistula: Three case reports and literature review. Neuroradiol J 2017;30:151-159.

16. Mayercik VA, Sussman ES, Pulli B, et al. Efficacy and safety of embolization of dural arteriovenous fistulas via the ophthalmic artery. Interv Neuroradiol 2021;27:444-450.

17. Meila D, Grieb D, Greling B, et al. Endovascular treatment of head and neck arteriovenous malformations: long-term angiographic and quality of life results. J Neurointerv Surg 2017;9:860-866.

18. Mukherjee B, Vijay V, Halbe S. Combined approach to management of periocular arteriovenous malformation by interventional radiology and surgical excision. Indian J Ophthalmol 2018;66:151-154.

19. Orlowski P, Al-Senani F, Summers P, Byrne J, Noble JA, Ventikos Y. Towards treatment planning for the embolization of arteriovenous malformations of the brain: intranidal hemodynamics modeling. IEEE Trans Biomed Eng 2011;58:1994-2001.

20. Park KB, Do YS, Kim DI, et al. Endovascular treatment results and risk factors for complications of body and extremity arteriovenous malformations. J Vasc Surg 2019;69:1207-1218. 
21. Rosenblatt TR, Myung D, Fischbein NJ, Steinberg GK, Kossler AL. Microsurgical Resection of an Orbital Arteriovenous Malformation With Intraoperative Digital Subtraction Angiography. Ophthalmic Plast Reconstr Surg 2021;37:S141-S144.

22. Settecase F, Hetts SW, Nicholson AD, et al. Superselective Intra-Arterial Ethanol Sclerotherapy of Feeding Artery and Nidal Aneurysms in Ruptured Cerebral Arteriovenous Malformations. AJNR Am J Neuroradiol 2016;37:692-697.

23. Shaver J. Eyelid arteriovenous malformation treated with embolization leading to a branch retinal artery occlusion. Optometry 2011;82:744-750.

24. Soulez G, Gilbert Md Frcpc P, Giroux Md Frcpc MF, Racicot Md Frcpc JN, Dubois J. Interventional Management of Arteriovenous Malformations. Tech Vasc Interv Radiol 2019;22:100633.

25. Su LX, Jia RB, Wang DM, Lv MM, Fan XD. Absolute ethanol embolization of arteriovenous malformations in the periorbital region. Cardiovasc Intervent Radiol 2015;38:632-641.

26. Terada T, Kinoshita Y, Yokote H, et al. Preoperative embolization of meningiomas fed by ophthalmic branch arteries. Surg Neurol 1996;45:161-166.

27. Thiex R, Wu I, Mulliken JB, Greene AK, Rahbar R, Orbach DB. Safety and clinical efficacy of Onyx for embolization of extracranial head and neck vascular anomalies. AJNR Am J Neuroradiol 2011;32:1082-1086.

28. Trivelatto F, Nakiri GS, Manisor M, et al. Preoperative onyx embolization of meningiomas fed by the ophthalmic artery: a case series. AJNR Am J Neuroradiol 2011;32:1762-1766.

29. Yoon YS, Ahn JY, Chang JH, et al. Pre-operative embolisation of internal carotid artery branches and pial vessels in hypervascular brain tumours. Acta Neurochir (Wien) 2008;150:447-452; discussion 452.

\section{Tables}

Table 1. Prior treatments/procedures and presenting signs and symptoms of 26 AVM patients

\begin{tabular}{llllll}
$\begin{array}{l}\text { Previous } \\
\text { therapy }\end{array}$ & $\begin{array}{l}\text { No. of patients } \\
(\%)\end{array}$ & Symptoms and signs & $\begin{array}{l}\text { No. of patients } \\
(\%)\end{array}$ & Location & $\begin{array}{l}\text { No. of patients } \\
(\%)\end{array}$ \\
\hline ISR+PAL & $11(42.3)$ & Pain & $7(26.9)$ & Right eyelid & $6(23.1)$ \\
\hline ISR+PAE & $2(7.7)$ & $\begin{array}{l}\text { Visual acuity } \\
\text { impairment }\end{array}$ & $11(42.3)$ & Left eyelid & $2(7.7)$ \\
\hline PAE & $7(26.9)$ & Visual field impairment & $8(30.7)$ & Left orbital & $6(23.1)$ \\
\hline Sclerotherapy & $6(23.1)$ & $\begin{array}{l}\text { Diplopia and } \\
\text { exophthalmos }\end{array}$ & $10(38.4)$ & Right orbital & $2(7.7)$ \\
\hline & Cosmetic deformities & $26(100)$ & Left & $5(19.2)$ \\
\hline & & Bleeding & $12(46.1)$ & Right nasal & $3(11.5)$ \\
\hline
\end{tabular}

Table 2 Results of the quality-of-life questionnaire 


\begin{tabular}{|c|c|c|c|c|c|c|c|}
\hline & & After treatme & & & & & \\
\hline Symptoms & $\begin{array}{l}\text { Before } \\
\text { treatment }\end{array}$ & $\begin{array}{l}\text { Complete } \\
\text { relief }\end{array}$ & $\begin{array}{l}\text { Major } \\
\text { improvement }\end{array}$ & $\begin{array}{l}\text { Minor } \\
\text { improvement }\end{array}$ & No relief & Worsening & $\mathrm{p}$ \\
\hline Pain & $7 / 26(26.9 \%)$ & $6 / 7(85.7 \%)$ & $1 / 7(14.25)$ & $0 / 7(0)$ & $0 / 7(0)$ & $0 / 26(0)$ & 0.031 \\
\hline $\begin{array}{l}\text { Visual acuity } \\
\text { impairment }\end{array}$ & $11 / 26(42.3 \%)$ & 4/11(36.6\%) & $3 / 11(27.2 \%)$ & $3 / 11(27.2 \%)$ & $1 / 11(9.0 \%)$ & $0 / 26(0)$ & 0.015 \\
\hline $\begin{array}{l}\text { Visual field } \\
\text { impairment }\end{array}$ & $8 / 26(30.7 \%)$ & $4 / 8(50.0 \%)$ & $2 / 8(25.0 \%)$ & $1 / 8(12.5 \%)$ & $1 / 8(12.5 \%)$ & $0 / 26(0)$ & 0.021 \\
\hline $\begin{array}{l}\text { Diplopia and } \\
\text { exophthalmos }\end{array}$ & $10 / 26(38.4 \%)$ & $2 / 10(20 \%)$ & $6 / 10(60.0 \%)$ & $2 / 10(20.0 \%)$ & $0 / 10(0)$ & $0 / 26(0)$ & 0.001 \\
\hline $\begin{array}{l}\text { Cosmetic } \\
\text { deformities }\end{array}$ & $26 / 26(100 \%)$ & 17/26(65.3\%) & 8/26(30.7\%) & $1 / 26(3.8 \%)$ & $0 / 26(0)$ & $0 / 26(0)$ & 0.001 \\
\hline $\begin{array}{l}\text { Impairment in } \\
\text { daily life }\end{array}$ & $26 / 26(100 \%)$ & $21 / 26(46.1 \%)$ & $5 / 26(19.2 \%)$ & $0 / 26(0)$ & $0 / 26(0)$ & $0 / 26(0)$ & 0.001 \\
\hline Bleeding & $12 / 26(46.1 \%)$ & $12 / 12(100 \%)$ & $0 / 12(0)$ & $0 / 12(0)$ & $0 / 12(0)$ & $0 / 26(0)$ & 0.001 \\
\hline
\end{tabular}

$p<0.05$ was considered statistically significant.

\section{Figures}



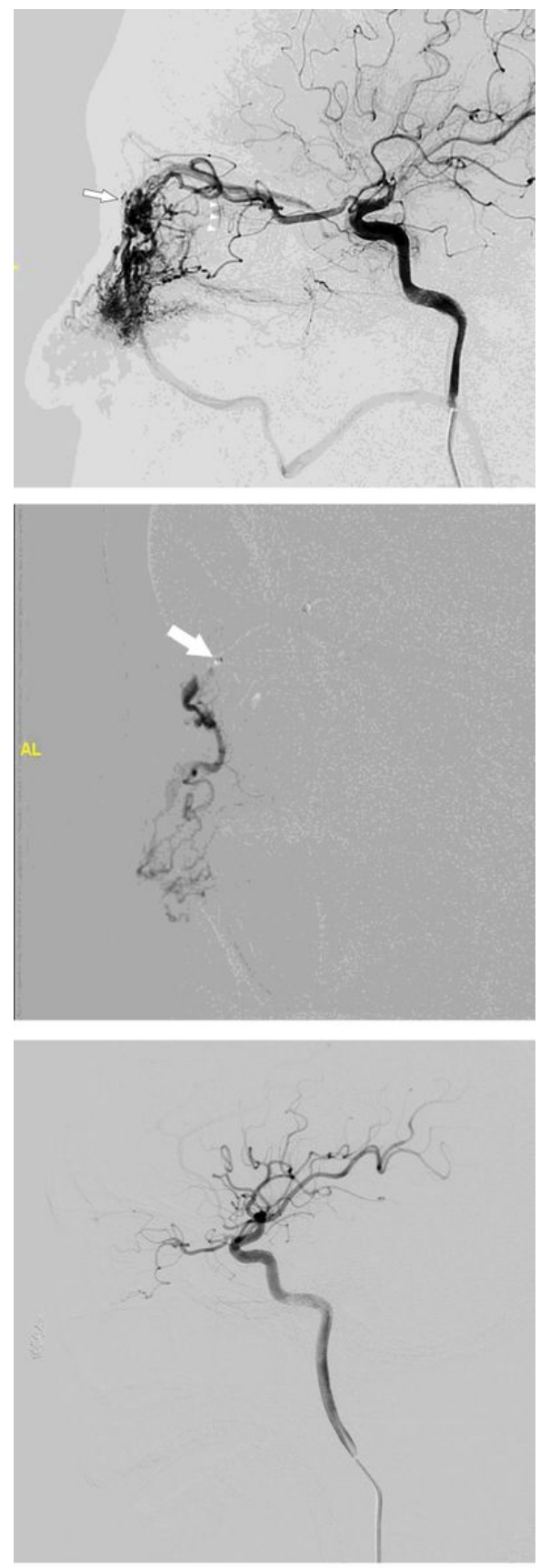

\section{Figure 1}

Patient with nose arteriovenous malformation presenting with mass/disfigurement and epstasis. (A) Internal carotid angiogram in the lateral view shows fistula and venous drainage (arrow) fed mainly by ophthalmic artery branches of OphA. Arrowheads delimitate the location of the choroidal crescent. Safety point (arrow) and ideal point (double arrow) are indicated. Tip of the microcatheter (arrow) in Ophthalmic artery was placed into draining veins as near the nidus as possible. First treatment session comprised transOphA embolization with ethanol (B). Patient returned after one month for a second session that comprised transOphA embolization with ethanol of residual lesions (data not shown). (C) Angiogram of arterial phase 12 months after embolization showing complete obliteration of AVM. Symptoms of the patient improved significantly. Patient reported no focal pain or discomfort at 12-months follow-up. 

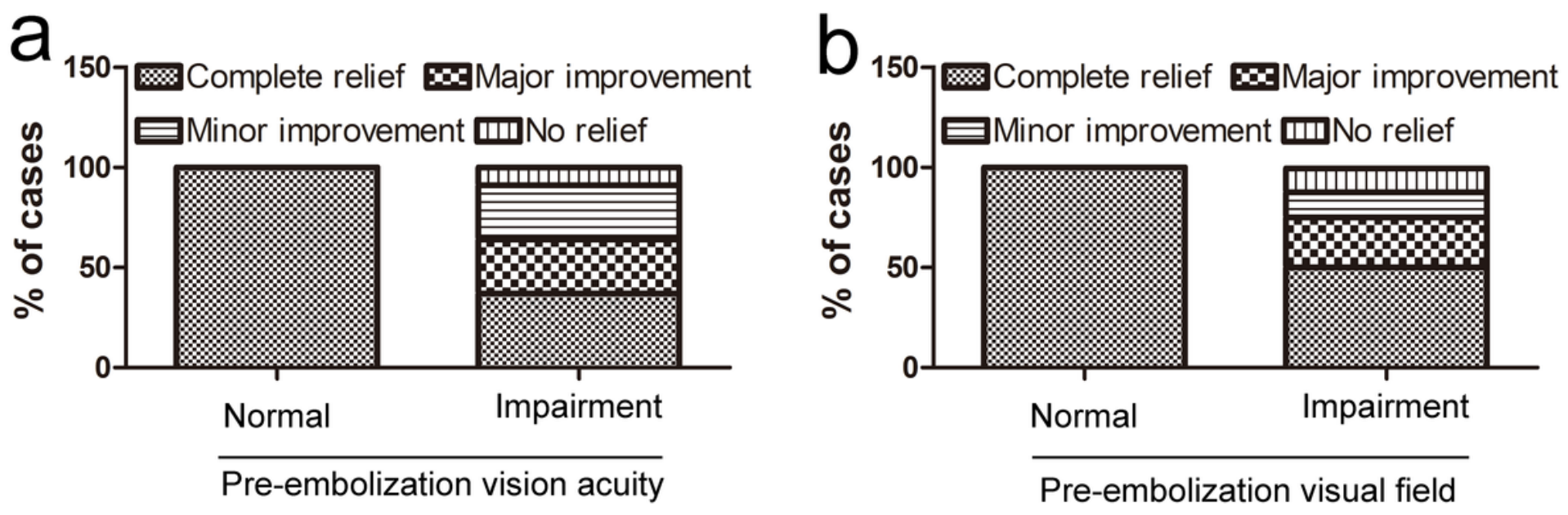

Figure 2

a In patients with preoperative impaired vision acuity, $63.6 \%$ showed significant improvement while all patients without preoperative visual deficit remained equal $(p<0.01)$. b Out of all patients with visual field impairment, $75 \%$ improved and all patients without preoperative visual field impairment remained unchanged $(p<0.01)$. 